HEP-LAT Preprint: hep-lat/9209007 Liverpool Preprint: LTH 284

Edinburgh Preprint: 92/511

August 27, 1992

\title{
SU(2) Potentials from large Lattices
}

\author{
UKQCD Collaboration
}

\section{S.P. Booth}

Department of Physics, The University of Edinburgh, Edinburgh EH9 3JZ, Scotland

A. Hulsebos, A.C. Irving, A. McKerrell, C. Michael, P.S. Spencer, P.W. Stephenson DAMTP, University of Liverpool, Liverpool L69 3BX, UK

\begin{abstract}
We measure accurate values of the inter-quark potentials on a $48^{3} 56$ lattice with $\mathrm{SU}(2)$ pure gauge theory at $\beta=2.85$. The scale is set by extracting the string tension - we obtain $\sqrt{K} a=0.063(3)$ at $\beta=2.85$. From a careful study of the small- $R$ potentials in the region $2 \mathrm{GeV}$ $<R^{-1}<5 \mathrm{GeV}$, we extract a running coupling constant and estimate the scale $\Lambda_{\overline{\mathrm{MS}}}=272(24) \mathrm{MeV}$.
\end{abstract}

\section{Introduction}

The only efficient non-perturbative technique at present for theories such as QCD is lattice simulation. It is important to demonstrate that the limit as the lattice spacing goes to zero is precisely understood. Moreover, the physical volume simulated should be kept constant as this spacing is reduced to disentangle scaling violation from finite size effects. This programme inevitably implies the study of lattices with many degrees of freedom and this is only feasible with recent increases in computing power.

To study this continuum limit in a non-abelian theory, we choose $\mathrm{SU}(2)$ pure gauge so that we can use the largest number of lattice sites. This work is a continuation of that reported [1] previously which was carried out using a Meiko Computing Surface composed of an array of $64 i 860$ processors with 
16 Mbytes of memory per processor. The results presented here are from our final analysis which yields more precise values for the interquark potential.

We also studied glueball and torelon spectra and our results (with relatively large errors) are also reported here.

The questions addressed in this paper are:

- Scaling: whether ratios of physical quantities are found to be independent of the lattice spacing $a$.

- The link to perturbation theory: whether lattice observables can be accurately matched to perturbation theory calculations so enabling the scale $\Lambda_{\overline{\mathrm{MS}}}$ to be determined in terms of non-perturbative quantities such as the string tension $K$.

\section{Updating $\mathrm{SU}(2)$ gauge fields}

We use the usual local algorithms for simulating pure $\mathrm{SU}(2)$ lattice gauge theory on a hypercubic lattice with periodic boundary conditions. The largest size of lattice that will fit in memory, while allowing for efficient inter-processor communications, is $48^{3} 56$. We estimate that we will be in the large volume region for such a lattice at $\beta$ values up to $\beta=2.85$, so we choose this value to be closest to the continuum limit. Previous work [2] has shown the virtues of using an over-relaxation algorithm (with $\omega=2$ ) to reduce the correlation between successive configurations. This is very efficient to implement in the case of $\mathrm{SU}(2)$ and we are able to obtain an update time of 458 nsec per link. For the heat-bath algorithm, we implement a variant of the Kennedy-Pendleton method [3] using a gaussian number generator based on Ahrens and Dieter's FL5 algorithm [4]. By coding in $i 860$ assembler, we obtain an update time per link of 635 nsec where each link is updated (so any link unaccepted is retried until accepted). Note that these update times are about 7 times faster than the fastest so far reported which came from [2] using a very efficient program on a CRAY-2.

We equilibrate our lattice configuration at $\beta=2.85$ with 1200 heat-bath sweeps. We then use a ratio of 4 over-relaxation to 1 heat-bath sweep with configurations saved for measurement every 300 sweeps. Previous results [2] at $\beta=2.7$ and 2.9 yield autocorrelation lengths of less than 300 sweeps for this updating procedure so we expect our configurations to be statistically independent. Subsequent analysis of our results for the potential $V(R)$ at large $R$ and for the glueball correlations confirms that the autocorrelation 
time is less than 450 sweeps for all observables. Our present results derive from 199 configurations - where several independent starts had to be made (in each case with 1200 heatbath equilibration sweeps). We evaluated the average plaquette action $S$ on our equilibrated configurations and obtain $S=0.70571(5)$. The potential and glueball measurements were computed on a CRAY XMP416 at RAL.

\section{Static quark - antiquark potentials}

The potential between static colour sources at separation $R$ can be obtained by measuring Wilson loops of size $R \times T$. Several methods are known to improve the accuracy of extraction of the ground state potential from this information. Thus a variational path basis allows excited states to be separated more easily, a fuzzing or blocking algorithm enhances the ground state signal, and $T$-extrapolation techniques of varying sophistication can be used.

\subsection{Variational path bases}

As just mentioned, it is useful to consider generalised correlations of size $R \times T$ where the spatial paths are any gauge invariant path with cylindrical symmetry $\left(A_{1 g}\right)$ - not just the straight line used in a rectangular Wilson loop. Moreover these two spatial paths, at times $t=T a$ apart, may be different. Hence the measurable will be $C_{i j}(R, T)$ where $i, j$ label the path type. Then the largest eigenvalue $\lambda(R)$ of the transfer matrix in the presence of the static sources is related to the potential by

$$
a V(R)=-\log (\lambda(R))
$$

and $\lambda(R)$ can be obtained from considering the limit as $T \rightarrow \infty$ of the largest eigenvalue $\lambda(R, T)$ of the equation

$$
C_{i j}(R, T) u_{j}=\lambda(R, T) C_{i j}(R, T-1) u_{j}
$$

This is a variational method where an optimum combination of paths $i$ (with $i=1, . . N)$ is chosen. At larger $T$, statistical errors may cause the eigenvalue analysis to become unstable. Thus a more robust procedure is to extract the optimum path basis $u_{i}$ from modest $T$-values such as $2 / 1$ and then extract 
the appropriate path combination $\mathcal{C}$ for study of larger $T$ :

$$
\mathcal{C}(R, T)=u_{i} C_{i j}(R, T) u_{j}
$$

and effective eigenvalue and potential values defined by

$$
\lambda_{\text {eff }}(R, T)=\frac{\mathcal{C}(R, T)}{\mathcal{C}(R, T-1)}=\exp \left(-V_{\text {eff }}(R, T) a\right)
$$

The best choice of paths would allow $\lambda_{e f f}(R, T)$ to be close to its asymptotic value for small $\mathrm{T}$. In this way the statistical errors will be smallest.

\section{$3.2 \quad$ Fuzzing}

As a measure of the efficiency of a path basis in extracting the ground state, it is convenient to introduce an "overlap" defined as

$$
\mathcal{O}(R)=\prod_{T}\left\{\lambda_{e f f}(R, T) / \lambda(R)\right\} .
$$

For straight paths of length $R / a=24$, we find $\mathcal{O}<0.05$. Thus rectangular Wilson loops are a very inaccurate way of extracting the ground state potential. As has been known for some time, a valuable improvement comes from fuzzing or blocking these paths [5, 6, 7]. Using a purely spatial blocking allows the transfer matrix interpretation to be retained. In order to reduce the memory and storage requirements of our lattice configurations, we performed one step of Teper blocking [5] which gives an effective spatial lattice of size $24^{3}$. We did not vary the parameters of this initial blocking step, but chose equal weights for the 5 paths (double straight path and $4 U$-bends) as found to be satisfactory previously [5, 7]. Because of this initial data smoothing we can only evaluate potentials at separations an even distance apart. For further smearing we used a more flexible scheme with no factor of two scale change: namely the recursive blocking scheme [6]:

$$
U(\text { new })=\mathcal{P}_{S U(2)}\left[c U(\text { straight })+\sum_{1}^{4} U(\text { u-bends })\right]
$$

After exploratory studies to optimise the overlap $\mathcal{O}$, we chose $c=2$ and a maximum of 110 iterations of this recursive blocking. Larger $c$ values gave slightly bigger overlaps but at the expense of much larger iterations (eg. 150 iterations at $c=4$ ). As a variational basis with $N=3$, we use 110, 70 and 30 iterations for the paths. This basis for $R / a=24$ yields an 
overlap $\mathcal{O}=0.92$ which is a huge improvement over the purely unblocked case. Because this value of the overlap is close to 1.0, one can get reliable estimates of the ground state potential from relatively small $T$-values which have smaller errors.

\subsection{T-extrapolation}

In order to extract the ground state potential values, one has to extrapolate the effective eigenvalues $\lambda(R, T)$ to large $T$. This will be controlled by the eigenstates of the lattice transfer matrix with

$$
C_{i j}(R, T)=b_{i}^{0} b_{j}^{0} \lambda_{0}^{T}(R)+b_{i}^{1} b_{j}^{1} \lambda_{1}^{T}(R)+. .
$$

where for a given $R$-value, $\lambda_{0}$ is related to the ground state potential as $\exp (-V a)$ and $\lambda_{1} / \lambda_{0}=\exp (-\Delta V a)$ with $\Delta V$ the energy gap to the first excited state. Then the extrapolation of the effective eigenvalue will be controlled by the admixture of excited state contributions, since

$$
\lambda_{e f f}(T)=\frac{\lambda_{0}^{T+1}+b \lambda_{1}^{T+1}+. .}{\lambda_{0}^{T}+b \lambda_{1}^{T}+. .}=\lambda_{0}\left(1-b\left(\frac{\lambda_{1}}{\lambda_{0}}\right)^{T}\left(1-\frac{\lambda_{1}}{\lambda_{0}}+. . .\right)\right)
$$

Since we use the same path operator (the linear combination corresponding to the $2 / 1$ eigenvector with largest eigenvalue) at each end, we will have $b>0$ which ensures that $\lambda_{\text {eff }}(T)$ monotonically increases to the asymptotic value $\lambda_{0}$ or equivalently that $V_{\text {eff }}(R, T)$ monotonically decreases to the asymptotic value $V(R)$. Now the appropriate energy gap is that to the first excited state that couples to the operator used for the path of length $R$. To avoid contamination by other path representations of lower energy, one should use the most symmetric path operators (with an $A_{1 g}$ representation under the group $D_{4 h}$ ), then the energy gap $\Delta V$ will be given by the next such $A_{1 g}$ state. Previous work [8, 7] has already determined the energy difference $\Delta V$ between the first excited $A_{1 g}$ state and the ground state we are trying to isolate. Moreover this determination agrees with the string model value of $2 \pi / R$ which should be an appropriate estimate at large $R$. This implies that the problem of excited state contamination in the signal will be most important at large $R$ where the energy gap is least. However, even at large $R, \Delta V a \approx 0.2$ and then any substantial contamination of this excited state would be very easily detectable as a steady decrease of the effective potential value with increasing $T$. 


\subsection{Data analysis}

We have results from measurements of 199 configurations. We combine these into 39 blocks of 5 adjacent measurements. Each such a block covers 1500 update sweeps and we find no statistically significant evidence for any autocorrelation between blocks. There is a strong correlation between the measurements of different sized Wilson loops, however. We take this into account primarily by using a bootstrap analysis of statistical errors. Thus we choose 39 blocks randomly (with substitution) from the 39 available and for each such choice we repeat the analysis of any quantity of interest. The variance over many such random selections then gives the error distribution required.

To estimate the potential $a V(R)$, we proceed as outlined above. With our variational basis of 3 paths (from the 3 different fuzzing levels), we can estimate the ground state and first excited state eigenvalues and eigenvectors of the transfer matrix even at relatively low $T$-ratios such as $2 / 1$. Since the statistical error is here quite small, we use this determination of the eigenvectors to fix the linear combination of the paths to be used at larger $T$. We then obtain effective potential values for each adjacent $T$-ratio and these are given in Table 1. To extrapolate to large $T$, we use two different methods. As a first method we identify the start of the plateau: namely the $T$-value at which the $(T+1) / T$ and $T /(T-1)$ effective potential values are equal within errors. Because of the strong correlation of the errors on these two quantities, we perform a bootstrap error analysis of the difference to ascertain the start of the plateau when this difference is statistically consistent with zero. In the preliminary analysis of part of this data [1], we found that the $4 / 3 T$-ratio was sufficient to give the plateau value. Because of the larger statistics in this work, we find somewhat larger $T$-values are needed in some cases. Indeed we find that the effective potential from $5 / 4$ is always consistent with being equal within one standard deviation to the $6 / 5$ effective potential. Thus the plateau value can be identified with the $5 / 4$ $T$-ratio. Note that since the effective potential is monotonically decreasing with $T$, the $5 / 4$ estimate is strictly an upper bound.

Because the excited state contamination is expected to be relatively larger at larger $R$, as discussed above, we may expect that the potential is overestimated more at large $R$ so yielding a larger string tension value. In order to account for this, the other method we use is an attempt to extrapolate to $T=\infty$ using the information on the energy gap to the first excited state to stabilise this extrapolation. We are able to control this extrapola- 


\begin{tabular}{|c|c|c|c|c|c|c|}
\hline$R / a$ & $a V(R, 3)$ & $a V(R, 4)$ & $a V(R, 5)$ & $a V(R, 6)$ & $a V(R, 7)$ & $a V_{\infty}(R)$ \\
\hline 2 & $0.3542(1)$ & $0.3540(1)$ & $0.3538(1)$ & $0.3538(2)$ & $0.3536(3)$ & $0.3534(2)$ \\
4 & $0.4181(1)$ & $0.4178(1)$ & $0.4176(2)$ & $0.4179(2)$ & $0.4181(4)$ & $0.4169(4)$ \\
6 & $0.4461(1)$ & $0.4456(2)$ & $0.4454(3)$ & $0.4456(4)$ & $0.4458(5)$ & $0.4442(4)$ \\
8 & $0.4645(2)$ & $0.4639(2)$ & $0.4639(2)$ & $0.4640(3)$ & $0.4634(5)$ & $0.4619(5)$ \\
10 & $0.4791(2)$ & $0.4782(3)$ & $0.4780(3)$ & $0.4783(5)$ & $0.4782(7)$ & $0.4754(7)$ \\
12 & $0.4915(3)$ & $0.4905(4)$ & $0.4906(3)$ & $0.4909(5)$ & $0.4900(6)$ & $0.4866(8)$ \\
14 & $0.5028(4)$ & $0.5017(4)$ & $0.5009(4)$ & $0.5009(6)$ & $0.5013(9)$ & $0.4976(9)$ \\
16 & $0.5134(5)$ & $0.5122(5)$ & $0.5120(5)$ & $0.5123(6)$ & $0.5118(10)$ & $0.5076(10)$ \\
18 & $0.5234(5)$ & $0.5220(6)$ & $0.5211(6)$ & $0.5218(7)$ & $0.5196(10)$ & $0.5165(11)$ \\
20 & $0.5333(7)$ & $0.5314(7)$ & $0.5301(8)$ & $0.5302(10)$ & $0.5296(11)$ & $0.5239(14)$ \\
22 & $0.5428(8)$ & $0.5409(8)$ & $0.5399(9)$ & $0.5397(10)$ & $0.5361(11)$ & $0.5328(18)$ \\
24 & $0.5522(9)$ & $0.5502(9)$ & $0.5489(9)$ & $0.5496(11)$ & $0.5481(13)$ & $0.5415(20)$ \\
\hline
\end{tabular}

Table 1: The effective potentials at $\beta=2.85$ between static sources at separation $R$. Here $V(R, T)$ is determined from the $T /(T-1)$ ratio of correlations as described in the text. $V_{\infty}(R)$ is determined from extrapolating the $3 / 2$ and $4 / 3$ effective potential values as described in the text.

tion because we have an estimate of the energy gap to the first excited state and we know that the effective potential has a monotonic decrease with increasing $T$. Thus by assuming that the approach to asymptopia is as slow as possible (ie it is given entirely given by the lowest excited state), we can extract the maximum systematic error in the evaluation of the ground state potential. This is most probably an overestimate of the contribution of the first excited state since the variational analysis selects a path combination which is designed to separate out the contributions from the ground state and first excited state - and if this has been accomplished then $b=0$.

Fitting the effective potential values for $T$ from 2 to 7 , we find an acceptable fit (using the full covariance matrix) with the excited state energy gap fixed from the $2 / 1$ variational analysis. In fact this fit gives very similar results to solving exactly for the two parameters $\lambda_{0}$ and $b$ using the effective potentials from $3 / 2$ and $4 / 3$ only. These results are shown in table 1. One indeed sees evidence for a sizeable systematic error particularly at larger $R$. One feature worth noting is that the difference $d V$ between the plateau value $V(R, 5)$ and the extrapolated value $V_{\infty}(R)$ is itself not smooth with $R$. It should be possible to use the reasonable requirement of continuity versus $R$ to constrain the correction somewhat - we have not pursued this. 


\subsection{Off-axis potentials}

As well as the preceding high statistics method of obtaining the potential between sites an even number of lattice spacings apart (and along a lattice axis), we carried out a complementary study of potentials at off-axis separations. We choose vector separations $\left(R_{x}, R_{y}, R_{z}\right)$ with $0 \leq R_{i} \leq 3 a$. For the 2 and 3 dimensional paths, we sum over all 2 (6) symmetric routes along the edges of the 2 (3) dimensional hypercuboid. We used the full $48^{3}$ spatial configuration and constructed operators by repeated blocking of links with [6] $c=2$ and 100 iterations. This level of blocking is less than that described above but it is optimal for the relatively shorter paths considered here. Indeed at $R=2 a$ where a direct comparison can be made, we find overlaps of $99.5 \%$ here whereas only $97.9 \%$ with the previous fuzzing scheme. Because of the computational and data communication constraints, we carried out this small- $R$ measurement for 8 configurations only. Nevertheless, the statistical errors are very small. We processed these results using the method of identifying a plateau as described above and we found that the $4 / 3$ and $5 / 4 T$-ratio effective potentials were equal within errors in every case. These results are shown in table 2. In subsequent analyses, we used the $4 / 3 T$-ratio values.

\subsection{R-dependent fits}

We concentrate first on extracting a string tension value from our high statistics data. To avoid the influence of the form of the fit at smaller $R$, we fit the static potential for $R \geq 6 a$ with the conventional lattice-Coulomb plus linear term:

$$
V(R)=C-\frac{E}{R}+K R
$$

A lattice-Coulomb expression for the lattice one gluon propagator on an infinite lattice is used for $1 / R$ although in practice this is very close to $1 / R$ for $R \geq 6 a$. Since the potential values at nearby $R$-values are highly correlated, we take into account the full covariance matrix in minimising $\chi^{2}$. Using a fit to the previously determined values of $V(R)$ and taking into account correlations in errors between potentials at different $R$-values, we find the result of table 3. The fit is illustrated in fig 1. The errors on the potential values are somewhat less correlated when expressed as difference values related to the force $(V(R+a)-V(R-a)) / 2 a$ and these data are collected in table 1 to allow other fits to be considered. 


\begin{tabular}{|c|c|c|c|c|}
\hline$R_{x} / a$ & $R_{y} / a$ & $R_{z} / a$ & $a V(R, 4)$ & $a V(R, 5)$ \\
\hline 1 & 0 & 0 & $0.2597(1)$ & $0.2596(1)$ \\
1 & 1 & 0 & $0.3226(1)$ & $0.3225(1)$ \\
1 & 1 & 1 & $0.3489(2)$ & $0.3489(3)$ \\
2 & 0 & 0 & $0.3538(2)$ & $0.3537(2)$ \\
2 & 1 & 0 & $0.3699(2)$ & $0.3697(2)$ \\
2 & 1 & 1 & $0.3802(2)$ & $0.3805(3)$ \\
2 & 2 & 0 & $0.3917(3)$ & $0.3916(4)$ \\
2 & 2 & 1 & $0.3972(4)$ & $0.3976(5)$ \\
2 & 2 & 2 & $0.4083(5)$ & $0.4081(6)$ \\
3 & 0 & 0 & $0.3944(4)$ & $0.3942(5)$ \\
3 & 1 & 0 & $0.3998(4)$ & $0.3998(5)$ \\
3 & 1 & 1 & $0.4040(4)$ & $0.4043(4)$ \\
3 & 2 & 0 & $0.4105(5)$ & $0.4107(6)$ \\
3 & 2 & 1 & $0.4134(4)$ & $0.4137(6)$ \\
3 & 2 & 2 & $0.4207(5)$ & $0.4209(6)$ \\
3 & 3 & 0 & $0.4224(5)$ & $0.4221(7)$ \\
3 & 3 & 1 & $0.4246(5)$ & $0.4246(6)$ \\
3 & 3 & 2 & $0.4294(6)$ & $0.4295(8)$ \\
3 & 3 & 3 & $0.4358(7)$ & $0.4356(9)$ \\
\hline
\end{tabular}

Table 2: The potentials at $\beta=2.85$ between static sources at separation $\left(R_{x}, R_{y}, R_{z}\right) / a$. Here $V(R, T)$ is determined from the $T /(T-1)$ ratio of correlations.

\begin{tabular}{|c|c|c|c|}
\hline & $E$ & $K a^{2}$ & $\chi^{2} /$ d.o.f. \\
\hline$V(R, 5)$ & $0.247(7)$ & $0.00401(8)$ & $15.2 / 7$ \\
$V_{\infty}(R)$ & $0.247(14)$ & $0.00363(17)$ & $8.2 / 7$ \\
\hline
\end{tabular}

Table 3: The fitted parameters to the potential for $R \geq 6 a$. 


\begin{tabular}{|c|c|c|}
\hline$R / a$ & $a V(R+2 a, 5)-a V(R, 5)$ & $a V_{\infty}(R+2 a)-a V_{\infty}(R)$ \\
\hline 2 & $0.0638(2)$ & $0.0635(3)$ \\
4 & $0.0278(2)$ & $0.0273(5)$ \\
6 & $0.0185(2)$ & $0.0177(5)$ \\
8 & $0.0141(2)$ & $0.0134(5)$ \\
10 & $0.0125(2)$ & $0.0112(5)$ \\
12 & $0.0103(2)$ & $0.0110(7)$ \\
14 & $0.0111(4)$ & $0.0100(7)$ \\
16 & $0.0091(4)$ & $0.0088(7)$ \\
18 & $0.0090(4)$ & $0.0074(7)$ \\
20 & $0.0098(4)$ & $0.0089(9)$ \\
22 & $0.0090(4)$ & $0.0089(14)$ \\
\hline
\end{tabular}

Table 4: The potential differences at $\beta=2.85$ between static sources at separation $R$

From these fits, we see the expected feature that the extrapolated potential values yield a smaller string tension since the extrapolation has most impact at large $R$. A reasonable approach would be to use the difference between the two string tension values as an estimate of the systematic error. Indeed the $V(R, 5)$ values are upper bounds whereas the $V_{\infty}$ values are very much extreme lower estimates. So the true value of the string tension should lie between the values extracted from our two fits. Since the $V(R, 5)$ analysis has the smaller statistical errors, we quote $K=0.00401(8)(38)$. This is effectively a $10 \%$ error on $K$. Note that the $\chi^{2}$ value is poor for the fit to the $T$-ratio $5 / 4$ effective potential. This is part of a general phenomenon that at larger $T$-values the errors seem to be non-gaussian.

As well as the errors from statistics and from $T$-extrapolation, there may also be errors due to the choice of function $V(R)$ to fit. To be specific, we have fitted for $0.38<R \sqrt{K}<1.52$ with a simple two parameter fit to the force (potential differences). If there were $\log (R)$ terms present as well, then the string tension would be much harder to extract. The fitted value for the string tension enables us to set the scale by requiring $K=0.44 \mathrm{GeV}$, so yielding $a^{-1}=6.95(35) \mathrm{GeV}$. Relating $a$ to $\Lambda_{L}$ by the two-loop perturbative $\beta$-function then gives $\sqrt{K} / \Lambda_{L}=43.0(2.1)$ at $\beta=2.85$.

We also wish to understand the potential at all $R$ and we follow the method used in [9] to make a comprehensive fit to on- and off-axis potential 


\begin{tabular}{|c|c|c|c|}
\hline$A$ & $f$ & $K a^{2}$ & $B / a$ \\
\hline $0.238(7)$ & $0.63(3)$ & 0.00401 & $0.050(6)$ \\
\hline
\end{tabular}

Table 5: Fit to force for $R>a$.

values. The potential shows a lack of rotational invariance at small $R$. To lowest order this can be attributed to the difference $\delta G(R)$ between the lattice one gluon exchange expression and the continuum expression.

$$
\delta G(R)=\frac{4 \pi}{a} \int_{-\pi}^{\pi} \frac{d^{3} k}{(2 \pi)^{3}} \frac{e^{i k \cdot R / a}}{4 \sum_{i=1}^{3} \sin ^{2}\left(k_{i} / 2\right)}-\frac{1}{R}
$$

On a lattice, the next order of perturbation has been calculated [10] for $R$ values on-axis and the dominant effect is a change from the bare coupling to an effective coupling [11]. In that case, using the difference above but with an adjustable strength will correct for the small $R / a$ lack of rotational invariance. A test of this will be that a smooth interpolation to the off-axis potential value of $V(R)$ versus $R$ is obtained with this one free parameter.

We evaluate $\delta G(R)$ numerically using the limit of a very large lattice since we are not here concerned with long-range effects. Then we use the following empirical expression to fit for $R>a$,

$$
V(R)=C-\frac{A}{R}+\frac{B}{R^{2}}+K R-A f \delta G(R)
$$

Here $f=1$ and $B=0$ corresponds to the previous two parameter fit where $E=A$. Now we fix the parameter $K$ from the foregoing large $R$ analysis, and fit to the smaller $R$ data with the above expression. We do not have sufficient data samples to use the full covariance matrix, so we fit to the force between adjacent $R$-values using the data for $V(R, 4)$ of Table 2. We find $\chi^{2}$ per degree of freedom 9.7/14 which is acceptable. The fit parameters are shown in table 5. The good fit quality shows that the lack of rotational invariance is indeed fully accommodated by the rather simple minded model of one lattice-gluon exchange. This is illustrated in fig 2 where the ratio of fit to data versus $R$ is shown.

For our present purposes the detailed form of this fit at small $R$ is not relevant - what is needed is a confirmation that a good fit can be obtained. This then supports our prescription to correct the lattice artifacts responsible for the lack of rotational invariance. What is more difficult is to assign 
errors to this correction procedure. Since the correction coefficient $f$ is determined to a few per cent, the statistical errors are small. The fact that one parameter corrects all the off-axis points simultaneously is very encouraging.

So in conclusion, we find that the lattice artifact effects from lack of rotational invariance can be well accommodated by the one gluon exchange correction with $f=0.63(3)$ and hence the lattice potential data can be corrected for this effect explicitly. This conclusion agrees with that [9] at $\beta=2.7$ where $f=0.68(3)$ was obtained.

\section{$4 \quad$ Scaling}

These results can be compared with a similar analyses [7, [0] on $32^{4}$ lattices at $\beta=2.7$ and on $24^{4}$ lattices [12] at $\beta=2.4$ for which the potentials have been calculated extensively. A particularly explicit way to test scaling is to plot the force versus $R$ at different $\beta$-values and match the resulting curves to one universal curve. The advantage of using the force is that self-energy effects cancel. In order to expose the scaling (ie $a$-dependence), we construct from the lattice artifact corrected potentials the dimensionless ratio

$$
\alpha\left(\frac{R_{1}+R_{2}}{2}\right)=\frac{4}{3} R_{1} R_{2} \frac{V_{c}\left(R_{1}\right)-V_{c}\left(R_{2}\right)}{R_{1}-R_{2}}
$$

(the factor $4 / 3$ is just a convention here). It turns out that the error in using a finite difference in $R$ is negligible for this quantity in our subsequent use. The advantage of this quantity $\alpha(R)$ is that it is easy to visualise the scaling between different $\beta$-values since the $R$-axis only has to be scaled.

Now we have access to accurate data on the force at $\beta=2.4$ from ref [12] and 2.7 from ref [9]. We consider the common physical $R$-range $0.4<$ $R \sqrt{K}<1.6$ and determine the $R$-scaling factor at 2.4 and 2.7 needed to bring the data to overlap best the 2.85 results from table 1 . The comparison is shown in fig 3 where we have used the best fit ratios of lattice spacings: $a(2.4) / a(2.85)=4.12(2)$ and $a(2.7) / a(2.85)=1.58(1)$ (these errors do not include the systematic error from $T$-extrapolation at 2.85$)$. We see that a universal curve describes the data well. This is especially impressive given that the ratio of lattice spacings is as large as 4 . Thus scaling is well satisfied by the interquark potential for $\beta \geq 2.4$.

A more detailed study of the ratio $a(2.7) / a(2.85)$ is possible once scaling is accepted. The most reliable determination comes from the force at moderate $R$ : at small $R$ the lattice artifact corrections are too big while at 
large $R$ the $T$-extrapolation errors are large. We use $\alpha(R=5 a(2.85))$ as the best determined point. We obtain $0.4448(32)$ from the $5 / 4 T$-extrapolation and $0.4368(80)$ from $V_{\infty}$. The lattice artifact correction (using $f A=0.150$ from our fit of table 5) is -0.0090 . Thus we determine $0.436(8)$ where the error covers statistical, $T$-extrapolation and lattice artifact correction. The lattice artifact corrected fit [9] to the $\beta=2.7$ data gives an interpolation in terms of $r=R / a(2.7)$ :

$$
\alpha(r)=0.348-0.144 / r+0.01373 r^{2}
$$

Assuming that the errors on this expression are similar (ie $2 \%$ on $\alpha$ at $\alpha$ near $0.436)$, we obtain $r=3.12(13)$. Hence we obtain $a(2.7) / a(2.85)=1.60(6)$. This ratio agrees with that illustrated above but now all errors are included.

As found previously [1], we find that asymptotic scaling is not valid at these $\beta$ values. Indeed since two-loop asymptotic scaling implies $a(2.7) / a(2.85)=1.464$, the above $a$-ratios already exclude asymptotic scaling. Another, more traditional, way to get at this ratio is to compare the string tension results at the two $\beta$-values. This has the disadvantage that an extrapolation in $R$ is required but is useful since the string tension is easy to compare between different lattice analyses. In order to try and reduce the systematic errors in $T$-extrapolation, we use similar analyses at the two $\beta$-values. Thus fitting in the same $R$-range $(0.4<R \sqrt{K}<1.6)$ and (a) using $T$-ratio $5 / 4$ we get string tension $K a^{2}=0.0109(2)$ and $0.00401(8)$; while (b) using the extrapolated value from $4 / 3$ and $3 / 2$ ratios gives $0.0103(2)$ and $0.00363(17)$ respectively at $\beta=2.7$ and 2.85 . Thus we evaluate $a(2.7) / a(2.85)=1.65(5)$ from the respective string tension measurements.

These discrepancies with asymptotic scaling can be expressed usefully in terms of the $\beta$-function. The perturbative expression for the effective $\beta$-function is

$$
\frac{-\Delta \beta}{\Delta \ln a}=\frac{11}{3 \pi^{2}}\left[1+\frac{17 g^{2}}{44 \pi^{2}}+\ldots\right]
$$

which is 0.393 at $\beta=2.7$ and 0.392 at 2.85 . Thus an accurate determination of $a(2.7) / a(2.85)$ will give the required $\beta$-function. Using $a(2.7) / a(2.85)=$ $1.60(6)$ gives $-\Delta \beta / \Delta \ln a=0.319(25)$ which is only $81 \%$ of the two loop perturbative result.

Thus we conclude that asymptotic scaling of the string tension is not found up to $\beta=2.85$. Moreover this effective $\beta$-function from 2.7 to 2.85 is rather close to that found at larger lattice spacing (e.g. from a comparison of $\beta=2.5$ and $2.7:-\Delta \beta / \Delta \ln a=0.318(10)$ from a string tension determination [7, 9] and 0.310(6) from a MCRG analysis [2]). 


\section{$5 \quad$ Glueballs and Torelons}

We also have measured glueball and torelon correlations on our 199 configurations. The operators were those used by ref [7] at $\beta=2.7$ except that another iteration of fuzzing was employed. The error analysis comes from a bootstrap evaluation of 39 blocks of 5 configurations each (as in the potential case). The effective masses in the $1 / 0$ variational path basis are shown in table 6. We see restoration of rotational symmetry (ie $E^{+}$and $T_{2}^{+}$representations of $O_{h}$ are degenerate and form the 5-dimensional $J^{P}=2^{+}$state, etc). It is reasonable to take the $3 / 2 T$-ratio effective masses as an estimate with a systematic error given by the differences between the results from different $T$-ratios. To compare with the results at $\beta=2.7$, for the $3 / 2$ effective mass, we quote the ratio $a(2.7) / a(2.85)$ obtained: namely $1.70(16)$ for $0^{+}$, 1.57(8) for $2^{+}$and 1.68(9) for $\mathrm{A}(100)$ torelon. These ratios are consistent with each other and with the scaling found from the inter-quark potential in the previous section. For the heavier states, we also confirm the results found at $\beta=2.7$, in particular the signal for a $J^{P}=1^{-}$state (from the $T_{1}^{-}$ representation) which has exotic spin-parity.

From the $A(100)$ torelon energy, we can find the effective string tension $K_{48}$ of the colour flux loop which encircles the spatial boundary of length $L=48 a$. This is $K_{48} a^{2}=0.00360(15)$. Correcting for string fluctuation effects then allows an estimate of the string tension $K=K_{48}+\pi /\left(3 L^{2}\right)=$ $0.00405(15) a^{-2}$. This latter value is in excellent agreement with our result from analysis of the heavy quark potential.

\section{Running coupling}

In the continuum the potential between static quarks is known perturbatively to two loops in terms of the scale $\Lambda_{\overline{\mathrm{MS}}}$. For $S U(2)$ colour, the continuum force is given by

$$
\frac{d V}{d R}=\frac{3}{4} \frac{\alpha(R)}{R^{2}}
$$

with the effective coupling $\alpha(R)$ defined as

$$
\alpha(R)=\frac{1}{4 \pi\left[b_{0} \log \left(R \Lambda_{R}\right)^{-2}+\left(b_{1} / b_{0}\right) \log \log \left(R \Lambda_{R}\right)^{-2}\right]}
$$

where $b_{0}=11 / 24 \pi^{2}$ and $b_{1}=102 b_{0}^{2} / 121$ are the usual coefficients in the perturbative expression for the $\beta$-function and, neglecting quark loops in 


\begin{tabular}{|c|l|l|l|l|l|}
\hline Rep & $1 / 0$ & $2 / 1$ & $3 / 2$ & $4 / 3$ & $5 / 4$ \\
\hline$A_{1}^{+}$ & $0.336(11)$ & $0.252(9)$ & $0.229(12)$ & $0.194(17)$ & $0.196(18)$ \\
$E^{+}$ & $0.529(11)$ & $0.377(12)$ & $0.357(16)$ & $0.358(27)$ & $0.373(13)$ \\
$T_{2}^{+}$ & $0.533(8)$ & $0.393(12)$ & $0.350(16)$ & $0.311(19)$ & $0.343(24)$ \\
$A_{1}^{-}$ & $0.690(19)$ & $0.430(26)$ & $0.448(35)$ & $0.403(57)$ & $0.286(73)$ \\
$E^{-}$ & $0.744(13)$ & $0.505(16)$ & $0.465(30)$ & $0.428(35)$ & $0.340(65)$ \\
$T_{2}^{-}$ & $0.752(11)$ & $0.533(14)$ & $0.510(25)$ & $0.505(38)$ & $0.422(83)$ \\
$A_{2}^{+}$ & $0.834(18)$ & $0.588(36)$ & $0.620(53)$ & $0.687(144)$ & \\
$T_{1}^{+}$ & $0.976(15)$ & $0.626(27)$ & $0.591(57)$ & $0.534(71)$ & \\
$A_{2}^{-}$ & $1.224(41)$ & $0.713(47)$ & $0.602(108)$ & $0.649(183)$ & \\
$T_{1}^{-}$ & $0.993(18)$ & $0.677(21)$ & $0.654(54)$ & $0.640(88)$ & $0.694(167)$ \\
$\mathrm{A}(100)$ & $0.248(7)$ & $0.176(6)$ & $0.173(7)$ & $0.167(8)$ & $0.155(8)$ \\
$\mathrm{A}(110)$ & $0.452(16)$ & $0.301(19)$ & $0.268(21)$ & $0.267(21)$ & $0.243(31)$ \\
$\mathrm{A}(111)$ & $0.696(69)$ & $0.355(55)$ & $0.292(53)$ & $0.268(82)$ & $0.275(70)$ \\
\hline
\end{tabular}

Table 6: The effective glueball and torelon masses at $\beta=2.85$ determined from the $T /(T-1)$ ratio of correlations as described in the text.

the vacuum, $\Lambda_{R}=1.048 \Lambda_{\overline{\mathrm{MS}}}$ (note that there is an error in this numerical value as quoted in ref.[9]). Note that the usual lattice regularisation scale $\Lambda_{L}=0.05045 \Lambda_{\overline{\mathrm{MS}}}$.

It is now straightforward to extract the running coupling constant from our lattice potentials by using [9]

$$
\alpha\left(\frac{R_{1}+R_{2}}{2}\right)=\frac{4}{3} R_{1} R_{2} \frac{V_{c}\left(R_{1}\right)-V_{c}\left(R_{2}\right)}{R_{1}-R_{2}}
$$

where the error in using a finite difference is here negligible. Here $V_{c}(R)$ are the potentials corrected for lattice artifacts as discussed above. We assign a systematic error of $10 \%$ to this correction as an illustration. These values are shown in table 7 and are plotted in fig 4 versus $R \sqrt{K}$ where $K$ is taken from the fit - see table 3. The interpretation of $\alpha$ as defined above as an effective running coupling constant is only justified at small $R$ where the perturbative expression dominates. Also shown are the two-loop perturbative results for $\alpha(R)$ for different values of $\Lambda_{R}$.

The figure clearly shows a running coupling constant. Moreover the result is consistent with the expected perturbative dependence on $R$ at small $R$. There are systematic errors, however. At larger $R$, the perturbative two- 
loop expression will not be an accurate estimate of the measured potentials, while at smaller $R$, the lattice artifact corrections are relatively big. Setting the scale using $\sqrt{K}=0.44 \mathrm{GeV}$ implies $1 / a(2.85)=7.0 \mathrm{GeV}$, so $R<$ $3.5 a(2.85)$ corresponds to values of $1 / R>2 \mathrm{GeV}$. This $R$-region is expected to be adequately described by perturbation theory. Another indication that perturbation theory is accurate at such $R$-values is that $\Delta V_{c} / \Delta R$ at small $R$ is found to be very much greater than the non-perturbative value $K$ at large $R$.

The curves shown in fig 10 allow an estimate of the running coupling at small $R$. In practice, $\alpha(R)$ is numerically nearly equal to $\alpha_{\overline{\mathrm{MS}}}$ at a momentum scale $q=1 / R$. Thus $\alpha_{\overline{\mathrm{MS}}}$ can be read directly off fig $\theta$. Since the behaviour is consistent with two-loop perturbation theory, we may express this in the conventional way in terms of a $\Lambda$ parameter. We can try to make a best fit to this data, however the smallest $R$ point is suspect since the lattice artifact correction is presumably largest, and the larger $R$ points are progressively affected by non-perturbative components. Thus we prefer to quote a band of values which encompass our results: $a \Lambda_{R}=0.041(3)$. Expressed in the conventional way this gives $\sqrt{K} / \Lambda_{L}=32.1(2.8)$. This result is in agreement with that found by a similar analysis [9] at $\beta=2.7$ namely $31.9(1.7)$. That agreement provides further justification for our procedure for removing the lattice artifacts from the force at small $R$. Because of the smaller lattice spacing in our present work, we are now able to push to smaller $R$-values $(R \sqrt{K}=0.1)$. This gives more confidence that the two loop perturbative expression for the force is accurate and hence determines $\Lambda$ more reliably.

A different method of extracting the running coupling constant from a study of finite volume effects on the lattice has recently been used by Lüscher et al. 13]. They analyse a succession of small-volume lattices to track the behaviour of the running coupling into the perturbative region. As a measurable they use the response to a constant colour electric field. They are able to reach scales corresponding to $R \sqrt{K}=0.05$ which is smaller than that we can reach here with confidence. They study the lattice size $L / a$ needed to have constant renormalised coupling as $\beta$ varies from 2.5 to 3.0. From this they obtain $a(\beta)$ and their result is in close agreement with asymptotic (to 2 loops) scaling. This result is in contrast with the large volume analyses (see above) which find $a(\beta)$ to decrease significantly faster with increasing $\beta$. These two observations suggest that the approach to asymptotic scaling differs in small volumes from large ones.

If that is the case, then it is difficult for them to set the scale since the 


\begin{tabular}{|c|l|l|l|}
\hline$R / a$ & \multicolumn{1}{|c|}{$\Delta V / \Delta R$} & $\Delta V_{c} / \Delta R$ & \multicolumn{1}{|c|}{$\alpha(R)$} \\
\hline 1.2071 & $0.1464(2)$ & 0.1175 & $0.2216(3)(65)$ \\
1.5731 & $0.0826(4)$ & 0.0750 & $0.2451(13)(25)$ \\
1.8660 & $0.0187(9)$ & 0.0568 & $0.2623(40)(177)$ \\
2.1180 & $0.0686(4)$ & 0.0463 & $0.2762(22)(131)$ \\
2.3428 & $0.0473(8)$ & 0.0404 & $0.2948(57)(56)$ \\
2.6390 & $0.0306(5)$ & 0.0326 & $0.3007(42)(20)$ \\
2.9142 & $0.0159(15)$ & 0.0277 & $0.3136(172)(136)$ \\
3.0811 & $0.0172(19)$ & 0.0247 & $0.3123(245)(106)$ \\
3.2395 & $0.0276(9)$ & 0.0234 & $0.3279(126)(54)$ \\
3.3904 & $0.0267(22)$ & 0.0245 & $0.3747(333)(70)$ \\
3.5348 & $0.0165(28)$ & 0.0198 & $0.3290(473)(72)$ \\
3.6736 & $0.0212(19)$ & 0.0200 & $0.3599(337)(28)$ \\
3.9324 & $0.0180(5)$ & 0.0188 & $0.3868(98)(5)$ \\
4.1231 & $0.0144(33)$ & 0.0160 & $0.3720(779)(42)$ \\
4.2426 & $0.0163(24)$ & 0.0179 & $0.4425(591)(18)$ \\
4.3589 & $0.0147(6)$ & 0.0144 & $0.3917(163)(4)$ \\
4.6904 & $0.0129(4)$ & 0.0128 & $0.4174(140)(2)$ \\
\hline
\end{tabular}

Table 7: The force $\Delta V / \Delta R$ and lattice artifact corrected force $\Delta V_{c} / \Delta R$ at average separation $R$. The running coupling $\alpha(R)$ derived from the corrected force is shown as well. The second error shown on $\alpha$ is $10 \%$ of the lattice artifact correction.

largest lattice size $L_{8}$ used by [13] corresponds to $z=m\left(0^{++}\right) L_{8}=3.2$. However, for $z<5$, the glueball and string tension results are significantly different from those on a large lattice [14]. The effect of adding a constant colour electric field is unlikely to change this conclusion that $z<5$ is very different from the large volume domain. Indeed the simulations of [13] are in the $z$-region which is well described by the semi-analytic methods of Van Baal [15]. It would be interesting to extend those calculations to include a constant colour electric field and make a direct comparison with ref [13]. Indeed it might be expected that scaling close to two-loop perturbative scaling would then be found since the calculation leans heavily on perturbation theory and the (relatively) trivial vacuum structure of small volumes. 


\section{Conclusions}

We have explored the lattice simulation of $\mathrm{SU}(2)$ gauge theory with the smallest lattice spacing used so far in a large volume study. It is important to keep the volume sufficiently large to avoid pronounced finite lattice effects. Indeed $\sqrt{K} L>2$ is needed 114 . Here we have $\sqrt{K} L=3$. To give a clearer indication of our results we set the scale using $\sqrt{K}=0.44 \mathrm{GeV}$. Then our lattice spacing $a=0.14 \mathrm{GeV}=0.028 \mathrm{fm}$ while the spatial lattice size is 1.3 $\mathrm{fm}$.

We find excellent scaling of large volume physical quantities for $\beta \geq$ 2.4. Thus the force $d V(R) / d R$ is a universal function of $R$ over a range of lattice spacings varying by a factor of 4 . This, combined with evidence from glueball spectra, points to an excellent agreement with scaling for all physical measurables in pure gauge $\mathrm{SU}(2)$ simulation at large volume. Recent small volume simulations [13] suggest that the behaviour $a(\beta)$ may be different in that case for the $\beta$-range we have explored. This would imply that the approach to asymptotic scaling is finite size dependent.

We do not find agreement with two-loop perturbative asymptotic scaling. Indeed the effective $\beta$-function is only $80 \%$ of the perturbative expression evaluated in terms of the bare lattice coupling constant from $\beta=4 / g^{2}$. This deviation can be ascribed to the bad choice of the lattice bare coupling constant as an expansion parameter. Using instead the small- $R$ force to define a renormalised coupling constant, we find a running coupling which varies as the two-loop perturbative expression. We explore this coupling down to scales $R \sqrt{K}=0.1$ which corresponds to $R^{-1}=4.4 \mathrm{GeV}$. This is a sufficiently hard momentum that the perturbative expression should be reliable and the scale $\Lambda$ can be estimated. We find our running coupling results can be described by $\Lambda_{\overline{\mathrm{MS}}}=272(24) \mathrm{MeV}$.

This work thus establishes that lattice gauge theory simulation is a viable method to extract continuum results. Further decrease in lattice spacing $a$ beyond that reported here is not expected to lead to any change in our results for ratios of physical quantities. We have extended this approach to $\mathrm{SU}(3)$ [16] in the quenched approximation but the further extension to full QCD with dynamical fermions is still needed and is a considerable computational challenge.

\section{Acknowledgements}

The computations using the Meiko Computing Surface at Edinburgh were supported by SERC under grants GR/G 32779 and GR/H 49191, by 
the University of Edinburgh and by Meiko Ltd. The computations on the Meiko Computing surface at Liverpool were supported by SERC under grant GR/G 37132 and EC contract SC1 *CT91-0642. The computations using the CRAY were supported by SERC grant GR/H 01236. We acknowledge a useful correspondence with Martin Lüscher. 


\section{References}

[1] S.P. Booth et al (the UKQCD Collaboration), Phys. Lett. B 275 (1992) 424.

[2] K. Decker and P. de Forcrand, Nucl. Phys. B (Proc. Suppl) 17(1990) 567.

[3] A. Kennedy and B. Pendleton, Phys. Lett. 156B (1985) 393.

[4] J. Ahrens and U. Dieter, Math. J. of Comp. 27 (1973) 927.

[5] M. Teper, Phys. Lett. 183B (1987) 345.

[6] M. Albanese et al., Phys. Lett. 192B (1987) 163.

[7] S. J. Perantonis and C. Michael, Nucl. Phys. B(Proc. Suppl) 20 (1991) 177; J. Phys. G (in press).

[8] S. J. Perantonis, A. Huntley and C. Michael, Nucl. Phys. B326 (1989) 544

[9] C. Michael, Phys. Lett. 283B (1992) 103.

[10] U. Heller and F. Karsch, Nucl. Phys. B251 (1985) 254.

[11] P. Lepage and P. Mackenzie, Nucl. Phys. B (Proc. Suppl.) 20 (1991) 173.

[12] C. Michael, Nucl. Phys. B (Proc. Suppl) 26(1992) 417.

[13] M. Lüscher, R. Sommer, U. Wolff and P. Weisz , Cern preprint TH 6566/92, DESY preprint 92-096.

[14] C. Michael, G. A. Tickle and M. Teper, Phys. Lett. B 207 (1988) 313.

[15] J. Koller and P. van Baal, Nucl. Phys. B302 (1988) 1.

[16] UKQCD collaboration, in preparation. 
Figure 1: The force between static quarks versus separation $R$ at $\beta=2.85$. The force (see table 4 ) is defined as a finite difference $a[V(R+a)-V(R-$ $a)] / 2$. The fit to the force for $R \geq 6 a$ is shown by the curve. 
Figure 2: The ratio between the potential values from table 2 and the fit with parameters given in table 5. As well as this fit with a lattice one gluon exchange component, we show (open triangles) the result of not making this correction for lattice artifacts. 
Figure 3: $\quad$ The force at different $\beta$-values compared using $\alpha(R)=$ $\frac{4}{3} R^{2} \Delta V_{c}(R) / \Delta R$, where $V_{c}$ is the potential corrected for lattice artifacts. Data at $\beta=2.85$ are from table 1 (diamonds), at $\beta=2.7$ (triangles) from ref [9] and at $\beta=2.4$ (squares) from ref [12]. Scaling corresponds to multiplying the $R$-axis by a constant as described in the text. 
Figure 4: The effective running coupling constant from table 0 versus $R$ at $\beta=2.85$. The statistical (continuous) and systematic (dotted) errors are shown. The curves represent the evolution expected from the two loop beta function with $a \Lambda_{R}=0.044$ (dotted) and 0.038 (continuous). 